\title{
ДИНАМИКА КЛИНИКО-ЛАБОРАТОРНЫХПРОЯВЛЕНИЙ ТИРОИДНОГО СИНДРОМА У ГИПЕРТЕНЗИВНЫХ БОЛЬНЫХ ПЕРВИЧНЫМ ГИПОТИРЕОЗОМ НА ФОНЕ ЛЕЧЕНИЯ
}

Частота гипотиреоза среди популяции больных с артериальной гипертензией (АГ) составляет 3-4\% [2]. Частота АГ среди всех больных гипотиреозом колеблется от 15 до 50\% [11].

АГ при гипотиреозе характеризуется часто вторичным происхождением, сопровождается системным периферическим артериальным спазмом, признаками гиперволемии, задержкой натрия, низким пульсовым давлением и носит преимущественно диастолический характер [3].

Хорошо изученным и одним из перспективных направлений немедикаментозного лечения гипертензивной категории больных гипотиреозом является курсовое применение сеансов гипокси-гиперокситерапии (ГГТ) $[1,4,10]$, что позволяет через активацию NO-опосредованной эндотелий-зависимой вазодилатации благотворно влиять на тонус периферических артерий $[1,4]$. Вместе с тем влияние ГГТ на тироидный обмен у такой категории больных остается малоизученным [5-6].

Цель исследования заключалась в анализе динамики клинических и лабораторных проявлений тироидного синдрома у гипертензивных больных первичным гипотиреозом на фоне двух режимов лечения (только медикаментозного и аналогичного, но с ГГТ).

\section{МАТЕРИАЛ И МЕТОДЫ}

В исследование включены 95 больных первичным гипотиреозом в возрасте от 30 до 65 лет. Критериями включения в исследование были: среднетяжелое течение гипотиреоза, гипотиреоз как следствие/исход хронического аутоиммунного тиреоидита, АГ 1-2 стадии и 1-2 степени, развившаяся во времени после дебюта гипотиреоза, письменное согласие на добровольное участие в проекте. Критерии исключения - возраст до 30 и старше 65 лет, тяжелое течение микседемы, послеоперационный (вторичный) гипотиреоз, рак щитовидной железы в анамнезе или статусе, микседематозное сердце (микседе- матозная кардиопатия), АГ 3 стадии, иной (симптоматический) характер гипертензии, непереносимость компонентов лечебной программы, сахарный диабет.

Методом случайной выборки пациенты разделены на две однотипные группы наблюдения, статистически однородные по полу $\left(\chi^{\#}=0,4\right.$, $\mathrm{p}=0,66)$, возрасту $(\mathrm{t}=1,8, \mathrm{p}=0,41)$, длительности гипертензивного ( $\mathrm{t}=0,8, \mathrm{p}=0,85)$ и тироидного $(\mathrm{t}=2,3, \mathrm{p}=0,14)$ синдромов, первая из которых $(\mathrm{n}=48)$ получала только стандартную терапию (левотироксин, ингибитор АПФ/сартан, тиазидный/тиазидоподобный диуретик, продленный антагонист кальция, статин, антиагрегант). Вторая группа $(\mathrm{n}=47)$ дополнительно к такому же самому медикаментозному лечению получала сеансы ГГТ (гипокси-гипероксикатор«Тибет-4», «Newlife», Россия). ГГТ проводили ежеквартально по 20 дней каждый на протяжении 5 лет (всего 20 курсов).

Соотношение мужчин и женщин в группах больных составило 1:4-4,5 (табл. 1.). Патология щитовидной железы во времени опережала гипертензивный синдром, что указывает на вторичность АГ.

Дозировки тиреозамещающих препаратов, а также антигипертензивных средств и их комбинаций корректировали исходя из динамично изменяющейся клинической и лабораторной ситуации течения гипертензивного и тироидного синдромов.

Группа контроля состояла из 30 практически здоровых людей аналогичного пола и возраста.

Больным 2-ой групп перед началом курса ГГТ определяли индивидуальную чувствительность к гипоксии путем проведения 10-минутного гипоксического теста (вдыхание через маску газовой смеси, содержащей $12 \%$ кислорода) с ежеми-

(c) Г.А. Игнатенко, И.В. Мухин, Т.С. Игнатенко,

Н.Ю. Паниева, 2020

(c) Университетская Клиника, 2020 
Таблица 1.

Характеристика больных и здоровых

\begin{tabular}{|c|c|c|c|}
\hline \multirow[b]{2}{*}{ Показатели } & \multicolumn{2}{|c|}{ Больные $(\mathrm{n}=95)$} & \multirow{2}{*}{$\begin{array}{c}\text { Здоровые } \\
(\mathrm{n}=30)\end{array}$} \\
\hline & $\begin{array}{c}\text { группа } 1 \\
(\mathrm{n}=48)\end{array}$ & $\begin{array}{c}\text { группа } 2 \\
(\mathrm{n}=47) \\
\end{array}$ & \\
\hline Возраст, $(\mathrm{M} \pm \mathrm{m}$, годы $)$ & $51,4 \pm 0,5$ & $52,0 \pm 0,9$ & $51,7 \pm 0,3$ \\
\hline Пол (мужчины/женщины), абс. & $9 / 39$ & $9 / 38$ & $8 / 22$ \\
\hline \multicolumn{4}{|l|}{ Стадия АГ: } \\
\hline I & $5(10,4 \%)$ & $5(10,6 \%)$ & - \\
\hline II & $43(89,6 \%)$ & $42(89,4 \%)$ & - \\
\hline \multicolumn{4}{|l|}{ Степень АГ: } \\
\hline I & $13(27,1 \%)$ & $13(27,6 \%)$ & - \\
\hline II & $35(72,9 \%)$ & $34(72,3 \%)$ & - \\
\hline Длительность патологии щитовидной железы, (M×m, годы) & $4,3 \pm 0,6$ & $4,5 \pm 0,8$ & - \\
\hline Длительность АГ, (Mm, годы) & $3,5 \pm 0,2$ & $3,6 \pm 0,3$ & - \\
\hline
\end{tabular}

Примечание: различия между аналогичными показателями в группах больных статистически не достоверны; различия между аналогичными показателями у больных и здоровых статистически не достоверны.

нутным мониторированием ЧСС и насыщения гемоглобина кислородом $\left(\mathrm{SaO}_{2}\right)$. Всем пациентам проводили пробы для дальнейшего выбора оптимальной схемы лечения. Пробу с задержкой дыхания проводили в двух вариантах: задержка дыхания на вдохе (проба Штанге) и задержка дыхания на выдохе (проба Генча). Пробы оценивали по продолжительности времени задержки и по показателю реакции (ПР) ЧСС. ПР получали при отношении величины ЧСС после окончания пробы к исходной частоте пульса. Тест с задержкой дыхания на вдохе проводили следующим образом. Исходно у обследуемого дважды подсчитывали пульс за 30 сек. в положении стоя. Дыхание задерживалось на полном вдохе, который обследуемый делал после трех дыханий на 75\% глубины полного вдоха. На нос надевали зажим. Время задержки регистрировали по секундомеру. Тотчас после возобновления дыхания подсчитывали частоту пульса. По длительности задержки дыхания пробу оценивали следующим образом: менее 39 с - результат неудовлетворительный; 39-49 с - результат удовлетворительный; свыше 50 с - результат хороший/достаточный. ПР у здоровых людей не превышала 1,2. Более высокие его значения свидетельствуют о негативной реакции сердечно-сосудистой системы на гипоксию. Каждый сеанс состоял из 5 серий пятиминутного вдыхания гипоксической смеси и интервалов вдыхания комнатного воздуха (нормоксические интервалы) такой же длительности или гипероксической газовой смеси $\mathrm{O}_{2}$ (вместо нормоксической паузы, которая традиционно используется при проведении стандартной интервальной нормобарической гипокситерапии).
При неудовлетворительной пробе Штанге и пробы Генча, ПР более 1,2 тренировки начинали с подачи 15\%-14\% гипоксической смеси. При удовлетворительной пробе Штанге и пробы Генча тренировки начинали 13\%-12\% насыщения кислородом гипоксической смеси. При пробе Штанге и пробе Генча - «хорошо» тренировки начинаются с подачи $12 \%-11 \%$ гипоксической смеси.

Исследование концентрации гормонов щитовидной железы (св. Т3 - трийодтиронин свободный, св. Т4 - тироксин свободный, ТТГ) в сыворотке крови проводилось с использованием иммуноферментного анализа.

Статистическая обработка данных проводилась с использованием пакета программы для статистического анализа «Statistica 6.0». Hopмальность распределения анализировали в тесте Колмогоров-Смирнова. Для сопоставления количественных параметров использовали критерий Стьюдента (t) для зависимых/независимых выборок. За уровень значимости (p) принимали величину $\mathrm{p}<0,05$.

\section{РЕЗУЛЬТАТЫ И ОБСУЖДЕНИЕ}

Динамика клинических проявлений гипотиреоидного синдрома представлена в таблице 2. Следует отметить, что ряд клинических проявлений тироидного синдрома в группах наблюдения довольно интенсивно уменьшился под влиянием терапии. К таковым можно отнести утомляемость (-30,9 и 49,9\% соответственно), отечность языка (-11,4 и 17,0\% соответственно), депрессия (-39,3 и 42,9\% соответственно), ощущение зябкости (-14,4 и 22,3\% соответственно), гиперкератоз (-10,0 и 16,5\% соответственно), ги- 
Динамика клинических проявлений тироидного синдрома у гипер-

Таблица 2. тензивных больных первичным гипотиреозом

\begin{tabular}{|c|c|c|c|}
\hline \multirow{4}{*}{ Показатели } & \multirow{3}{*}{$\begin{array}{c}\text { Этапы обследования } \\
\text { I }\end{array}$} & \multicolumn{2}{|c|}{ Больные } \\
\hline & & группа 1 & группа 2 \\
\hline & & $(\mathrm{n}=48)$ & $(\mathrm{n}=47)$ \\
\hline & II & $(\mathrm{n}=38)$ & $(\mathrm{n}=43)$ \\
\hline \multirow{2}{*}{ Утомляемость } & $\mathrm{I}$ & $30(62,5 \%)$ & $30(63,8 \%)$ \\
\hline & II & $12(31,6 \%)^{\#}$ & $6(13,9 \%)^{\#}$ \\
\hline \multirow{2}{*}{ Рассеянность } & $\mathrm{I}$ & $14(29,2 \%)$ & $15(31,9 \%)$ \\
\hline & II & $10(26,3 \%)^{\#}$ & $10(23,3 \%)^{\#}$ \\
\hline \multirow{2}{*}{$\begin{array}{l}\text { Отечность языка } \\
\text { увеличение губ и языка }\end{array}$} & $\mathrm{I}$ & $8(16,7 \%)$ & $8(17,0 \%)$ \\
\hline & II & $2(5,3 \%)^{\#}$ & - \\
\hline \multirow{2}{*}{ Параорбитальные отеки } & I & $14(29,2 \%)$ & $14(29,8 \%)$ \\
\hline & II & $11(28,9 \%)$ & $10(23,3 \%)^{\#}$ \\
\hline \multirow{2}{*}{ Отсутствие жалоб } & $\mathrm{I}$ & $4(8,3 \%)$ & $3(6,4 \%)$ \\
\hline & II & $19(50,0 \%)^{\#}$ & $24(55,8 \%)^{* \#}$ \\
\hline \multirow{2}{*}{ Запоры } & $\mathrm{I}$ & $11(22,9 \%)$ & $11(23,4 \%)$ \\
\hline & II & $8(21,1 \%)$ & $8(18,6 \%)^{\#}$ \\
\hline \multirow{2}{*}{ Депрессия } & I & $29(60,4 \%)$ & $30(63,8 \%)$ \\
\hline & II & $20(21,1 \%)^{\#}$ & $9(20,9 \%)^{\#}$ \\
\hline \multirow{2}{*}{ Снижение памяти } & $\mathrm{I}$ & $24(50,0 \%)$ & $24(51,5 \%)$ \\
\hline & II & $19(50,0 \%)^{\#}$ & $16(37,2 \%)^{* \#}$ \\
\hline \multirow{2}{*}{ Зябкость } & $\mathrm{I}$ & $17(35,4 \%)$ & $17(36,2 \%)$ \\
\hline & II & $8(21,0 \%)^{\#}$ & $6(13,9 \%)^{* \#}$ \\
\hline \multirow{2}{*}{ Увеличение массы тела } & $\mathrm{I}$ & $35(72,9 \%)$ & $34(72,3 \%)$ \\
\hline & II & $31(81,6 \%)^{\#}$ & $30(69,7 \%)^{\#}$ \\
\hline \multirow{2}{*}{ Сухость кожи } & $\mathrm{I}$ & $12(25,0 \%)$ & $11(23,4 \%)$ \\
\hline & II & $6(15,8 \%)^{\#}$ & $3(6,9 \%)^{* \#}$ \\
\hline \multirow{2}{*}{ Гипотермия } & $\mathrm{I}$ & $5(10,4 \%)$ & $5(10,6 \%)$ \\
\hline & II & $2(5,3 \%)^{\#}$ & $2(4,6 \%)^{\#}$ \\
\hline
\end{tabular}

Примечание: I - до лечения, II - через 5 лет; * различия между аналогичными показателями в 1-ой и 2-ой группах статистически достоверны; \# - различия между аналогичными показателями на 1-м и 2-м этапах статистически достоверны.

потермия (-5,1 и 6,0\% соответственно). При этом достоверно увеличилась частота отсутствия жалоб (+41,7 и 49,4\% соответственно).

Аутоиммунные заболевания щитовидной железы представляют собой более сложную научную и клиническую проблему, чем йододефицитные. Риск развития аутоиммунной патологии на 80\% зависит от генетической предрасположенности. Вклад факторов окружающей среды, обеспечивающих реализацию этой предрасположенности, составляет около 20\% [7]. На практике врачи сталкиваются либо с субклиническим гипотиреозом аутоиммунной природы, либо, значительно реже, с классическими проявлениями гипотиреоза. Учитывая, что в современном мире основной причиной формирова- ния синдрома гипотиреоза являются хронические аутоиммунные тиреоидиты, которые протекают с невыраженной симптоматикой или бессимптомно, и диагностируются ретроспективно уже только на стадии утраты функциональной активности органа за счет тотального или субтотального фиброза.

Заместительная терапия тироидными гормонами при гипотиреозе проводится для выведения больного из микседематозного состояния и замены его эутиреоидным, при котором наступает нормализация нарушенных процессов обмена [8]. Критерием адекватности заместительного лечения является исчезновение симптомов гипотиреоза и нормализация гормональных показателей. 
Однократный прием левотироксина сохраняет биологически значимый уровень Т3 в течение суток благодаря периферической конверсии T4 в T3, максимальная его концентрация в крови определяется через 3-4 ч. Исходя из представления о том, что щитовидная железа продуцирует два гормона - тироксин и трийодтиронин, длительное время считалось, что для лечения гипотиреоза предпочтительнее использовать их комбинацию, чем монотерапию одним из препаратов [13]. Позже было показано, что приблизительно 80\% циркулирующего в крови трийодтиронина образуется за счет дейодирования тироксина в периферических тканях. Кроме того, прием препарата трийодтиронина сопровождается достижением пикового нефизиологического уровня с достаточно быстрым последующим его убыванием. Поэтому для заместительной терапии гипотиреоза предпочтительными являются препараты левотироксина. Выбор стартовой дозы зависит от следующих факторов: степени тяжести гипофункции, возраста пациента, наличия сопутствующей сердечно-сосудистой патологии. Лечение гипотиреоза у взрослых целесообразно начинать с небольшой дозы левотироксина с учетом того, что неадекватно высокая доза и ее быстрое наращивание приводит к повышению артериального давления [12]. В идеале препарат следует принимать в одно и то же время суток (утром) за 30-40 мин. до завтрака, запив его водой, как минимум с интервалом в 4 ч., до или после приема других лекарственных препаратов. Полная поддерживающая доза левотироксина у взрослых определяется из расчета 1,6 мкг на 1 кг массы тела и составляет в среднем для женщин 100 мкг/сут, для мужчин - 150 мкг/сут, для пожилых - 50 мкг/сут или менее[11].

у пациентов, страдающих сердечнососудистой патологией, длительность периода адаптации к левотироксину может составить 4-6 мес., а заместительная доза препарата не должна превышать 0,9 мкг/кг. В подавляющем большинстве случаев требуется пожизненное проведение заместительной терапии манифестного гипотиреоза [3].

Основные побочные эффекты терапии левотироксином связаны с неблагоприятным воздействием на течение сердечно-сосудистой патологии: появление/усугубление ишемии вплоть до инфаркта миокарда, повышение артериального давления, формирование гипертрофии левого желудочка, нарушения ритма, в т.ч. и фибрилляция предсердий и внезапная остановка сердца $[2,7]$.

Препараты, содержащие Т3, назначают несколько раз в день для поддержания нормаль- ного уровня гормона в крови, так как Т3 имеет короткий период полувыведения. После приёма Т3 (лиотиронин) отмечается быстрое и значительное повышение значений Т3, которые нормализуются через 2-4 часа [13]. У пациентов, получающих Т3 несколько раз в сутки, отмечается состояние медикаментозного тиреотоксикоза. У таких больных повышен риск возникновения сердечной патологии и АГ. Комбинированные препараты Т3 и Т4 (тиреокомб, тиреотом, новотирол, йодтирокс) используют по особым показаниям. При их приёме отмечают аналогичную динамику содержания Т3, хоть и с меньшими 25 пиковыми уровнями. Монотерапия L-тироксином вызывает плавное увеличение содержания в плазме Т3 в течение нескольких недель. Референтные значения достигаются в течение 8 недель терапии.

Эффективность лечения оценивают по клиническим признакам (частота пульса, уровень артериального давления, выраженность отеков, изменений кожи и нервной системы), по динамике ЭКГ и по результатам исследования уровня тиреотропного гормона (ТТГ), который снижается до нормы постепенно в течение 2-3 месяцев [9]. Если по истечении этого срока уровень ТТГ не нормализуется, увеличивают дозу левотироксина на 12,5-25 мкг каждые 2-4 недели [13]. Больным с ИБС или хроническими заболеваниями легких лечение начинают с малых доз (2550 мкг/сут) с последующим постепенным увеличением (на 25 мкг в месяц). Выбор такой тактики определяется тем, что миокард больных с гипотиреозом высокочувствителен к тиреоидным гормонам, а быстрое достижение эутиреоза приводит к значительному увеличению потребности миокарда в кислороде, что может привести к обострению ИБС и возникновению опасных для жизни аритмий [11-12]. Необходима большая осторожность при назначении тиреоидных гормонов лицам пожилого возраста, так как у многих пожилых больных имеется ИБС. Как правило, заместительную терапию начинают с низких доз (не более 50 мкг/сут, чаще - 12,5-25 мкг/сут). Наращивание дозы проводится очень медленно (на 25 мкг в 1-2 месяца). Повышение дозы возможно лишь при отсутствии стенокардии, повышения артериального давления и отрицательной динамики ЭКГ [3]. При появлении клинических или ЭКГ-признаков ухудшения коронарного кровообращения дозу тироксина уменьшают до предыдущей. В случае возникновения тахикардии или болей в области сердца для улучшения адаптации к тиреоидной терапии добавляют малые дозы бета-блокаторов [12]. С возрастом суточная потребность в тиреоидных гормонах снижается, у лиц старше 65 лет она состав- 
ляет в среднем 1,5 мкг/кг/сут. После достижения этой дозы дальнейший подбор осуществляют под контролем клиники, ЭКГ и данных гормональных исследований (ТТГ или Т4).

При субклиническом течении гипотиреоза вопрос о заместительной терапии решается индивидуально. Принимая во внимание высокую частоту дислипидемий, риск развития атеросклероза и инфаркта миокарда, можно считать оправданным назначение препаратов левотироксина натрия [7]. Прежде чем начать лечение, следует убедиться, что субклинический гипотиреоз не носит транзиторный характер. С этой целью рекомендуется повторить исследование ТТГ и св. Т4 через 3-6 месяцев. Цель лечения нормализация уровня ТТГ, что, как правило, достигается назначением тироксина в дозе не более 1 мкг/кг/сут, т. е. 50-75 мкг в день (в ряде случаев достаточно и 25-50 мкг/сут) [8].

Анализ динамики лабораторных маркеров функциональной активности щитовидной железы показал выраженное достоверное понижение уровня ТТГ в группе 2 до концентрации вещества, соответствующего уровню здоровых людей (табл. 3.). При этом концентрация ТТГ на 2-м этапе обследования не имела достоверных различий с группой здоровых людей. Прирост уровня Т3 св. в группах равнялся 0,42 и 0,68 нг/л соответственно. При этом концентрация Т3 св. на фоне терапии увеличилась преимущественно в группе 2, но так и не достигла нормального значения. Уровень Т4 св. вырос на 2,3 и 4,3 мкмоль/л соответственно, вплотную приблизившись к нормативным параметрам. Концентрация антител в крови снизилась, но в большей степени в группе 1. При этом различия между группами по величине АТТГ и АТПО равнялись 46,4 и 115,1 в пользу более высокого содержания в группе 2. Последнее может косвенно указывать на то, что в группе 1 вследствие активного аутоиммунного процесса темпы гибели тироидной ткани и замещения ее соединительной тканью были более высокие, чем в группе 2, где эти процессы происходили медленней. В этой связи в группе 1 мы имеем более интенсивное понижение уровня антител, чем в группе 2. При этом дополнительного экзогенного воздействия на процессы аутоиммунизации не происходило, поскольку пациенты не получали ни глюкокортикоидных гормонов, которые чаще назначают в подобных ситуация, ни тем более иммунодепрессантов. В любом случае достижения уровня антител ни в одной из групп не произошло.

\section{В Ы В О д Ы}

1.Исходные клинические проявления тироидного синдрома у гипертензивных больных первичным гипотиреозом характеризовались утомляемостью, параорбитальными отеками, депрессией, снижением памяти, ощущением зябкости, увеличением массы тела и сухостью кожи.

2. Комплексная медикаментозно-гипоксигиперокситерапия в группе 2 характеризова-

Таблица 3.

Динамика гормонов щитовидной железы и антитироидных антител у гипертензивных больных первичным гипотиреозом, а также у здоровых

\begin{tabular}{|c|c|c|c|c|}
\hline \multirow{4}{*}{ Показатели } & \multirow{3}{*}{$\begin{array}{c}\text { Этапы обследования } \\
\text { I } \\
\end{array}$} & \multicolumn{2}{|c|}{ Больные } & \multirow{4}{*}{ Здоровые } \\
\hline & & группа 1 & группа 2 & \\
\hline & & $(\mathrm{n}=48)$ & $(\mathrm{n}=47)$ & \\
\hline & II & $(n=38)$ & $(n=43)$ & \\
\hline \multirow{2}{*}{ ТТГ, мЕД/л } & $\mathrm{I}$ & $7,50 \pm 0,08^{\Delta}$ & $7,52 \pm 0,04^{\Delta}$ & \multirow{2}{*}{$1,88 \pm 0,03$} \\
\hline & II & $4,91 \pm 0,03^{\# \Delta}$ & $2,05 \pm 0,09^{* \#}$ & \\
\hline \multirow{2}{*}{ Т3 св., нг/л } & $\mathrm{I}$ & $2,44 \pm 0,01^{\Delta}$ & $2,47 \pm 0,03$ & \multirow{2}{*}{$4,50 \pm 0,07$} \\
\hline & II & $2,86 \pm 0,04^{\Delta}$ & $3,15 \pm 0,05^{* \# \Delta}$ & \\
\hline \multirow{2}{*}{ Т4 св., мкмоль/л } & I & $9,5 \pm 0,09^{\Delta}$ & $9,4 \pm 0,10$ & \multirow{2}{*}{$15,05 \pm 0,11$} \\
\hline & II & $11,8 \pm 0,04^{\Delta \sharp}$ & $13,7 \pm 0,07^{* \# \Delta}$ & \\
\hline \multirow{2}{*}{ АТТГ, Ед/л } & I & $207,0 \pm 2,11^{\Delta}$ & $205,8 \pm 2,45$ & \multirow{2}{*}{$12,2 \pm 1,16$} \\
\hline & II & $122,5 \pm 2,47^{\# \Delta}$ & $168,9 \pm 2,28^{* \# \Delta}$ & \\
\hline \multirow{2}{*}{ АТПО, Ед/л } & $\mathrm{I}$ & $708,5 \pm 2,08^{\Delta}$ & $710,3 \pm 2,14$ & \multirow{2}{*}{$6,9 \pm 0,05$} \\
\hline & II & $412,9 \pm 2,81^{\# \Delta}$ & $528,0 \pm 2,45^{* \# \Delta}$ & \\
\hline
\end{tabular}

Примечание: I - до лечения, II - через 5 лет; * - различия между аналогичными показателями в 1-ой и 2-ой группах статистически достоверны; \# - различия между аналогичными показателями на 1-м и 2-м этапах статистически достоверны; $\Delta$ - различия между аналогичными показателями у больных и здоровых статистически достоверны. 
лась значительно более интенсивным уменьшением частоты симптомов тироидного синдрома, чем стандартная терапия в группе 1 . Регресс симптомов тироидного синдрома в группе 2 на 2-м этапе обследования по сравнению с группой 1 касался таких проявлений, как: утомляемость $(-17,7 \%)$, ощущение зябкости $(-7,1 \%)$, увеличение массы тела $(-11,9 \%)$. При этом в группе 2 по сравнению с группой 1 на 5,8\% увеличилась численность бессимптомных пациентов, что свидетельствует о трансформации декомпенсированного гипотиреоза в фазу субкомпенсации или компенсации тироидного обмена.

3. Исходные лабораторные параметры тироидного синдрома свидетельствуют о формировании первичного гипотиреоза средней тяже- сти течения, развившегося на фоне хронического аутоиммунного тиреоидита.

4. Лечение в группе 2 способствовало интенсификации восстановления уровня тироидных гормонов на фоне угнетения синтеза ТТГ, что свидетельствует о медикаментозной компенсации тироидного обмена. Напротив, в группе 1 компенсации тироидного метаболизма не получено.

5. Интенсивное снижение уровней антитироидных антител в группе 1 может косвенно свидетельствовать о более активных процессах утраты функции щитовидной железы и вторичном снижении активности аутоиммунных реакций.

\section{Г.А. Игнатенко, И.В. Мухин, Т.С. Игнатенко, Н.Ю. Паниева}

ГОО ВПО «Донецкий национальный медицинский университет имени М. Горького», Донецк

ДИНАМИКА КЛИНИКО-ЛАБОРАТОРНЫХПРОЯВЛЕНИЙ ТИРОИДНОГО СИНДРОМА
У ГИПЕРТЕНЗИВНЫХ БОЛЬНЫХ ПЕРВИЧНЫМ ГИПОТИРЕОЗОМ НА ФОНЕ ЛЕЧЕНИЯ

Одним из перспективных направлений немедикаментозного лечения такой категории больных является курсовое применение сеансов гипоксигиперокситерапии, что позволяет через активацию $\mathrm{NO}$-опосредованной эндотелий-зависимой вазодилатации благотворно влиять на проявления гипертензивного синдрома. Вместе с тем влияние гипоксигиперокситерапии на тироидный обмен у такой категории больных остается малоизученным.

Цель исследования заключалась в анализе динамики клинических и лабораторных проявлений тироидного синдрома у гипертензивных больных первичным гипотиреозом на фоне двух режимов лечения (только медикаментозного и аналогичного, но с гипокси-гиперокситерапией).

В исследование включены 95 больных первичным гипотиреозом в возрасте от 30 до 65 лет. Методом случайной выборки пациенты разделены на две однотипные группы наблюдения, статистически гомогенные по полу, возрасту, длительности гипертензивного и тироидного синдромов, первая из которых $(\mathrm{n}=48)$ получала только стандартную терапию (левотироксин, ингибитор ангиотензинпревращающего фермента/сартан, тиазидный/тиазидоподобный диуретик, продленный антагонист кальция, статин, антиагрегант). Вторая группа $(\mathrm{n}=47)$ дополнительно к такому же самому медикаментозному лечению получала сеансы гипокси-гиперокситерапии (гипокси-гипероксикатор «Тибет-4», «Newlife», Россия). Гипокси-гиперокситерапию проводили ежеквартально по 20 дней каждый на протяжении 5 лет (всего 20 курсов).

По результатам исследования исходные клинические проявления тироидного синдрома у гипертензивных больных первичным гипотиреозом характеризовались превалированием утомляемости, параор- битальных отеков, депрессией, снижением памяти, ощущением зябкости, увеличением массы тела и сухостью кожи.

Комплексная медикаментозно-гипокси-гиперокситерапия в группе 2 характеризовалась значительно более интенсивным уменьшением частоты симптомов тироидного синдрома, чем стандартная терапия в группе 1. Регресс симптомов тироидного синдрома в группе 2 на 2-м этапе обследования по сравнению с группой 1 касался таких проявлений, как: утомляемость $(-17,7 \%)$, ощущение зябкости $(-7,1 \%)$, увеличение массы тела (-11,9\%). При этом в группе 2 по сравнению с группой 1 на 5,8\% увеличилась численность бессимптомных пациентов, что свидетельствует о трансформации гипотиреоза в фазу суб-/компенсации тироидного обмена.

Исходные лабораторные параметры тироидного синдрома свидетельствуют о формировании первичного гипотиреоза средней тяжести течения, развившегося на фоне хронического аутоиммунного тиреоидита.

Лечение в группе 2 способствовало интенсификации восстановления уровня тироидных гормонов на фоне угнетения синтеза тиреотропного гормона, что свидетельствует о медикаментозной компенсации тироидного обмена. Напротив, в группе 1 компенсации тироидного метаболизма не получено.

Более интенсивное снижение уровней противотироидных антител в группе 1 может косвенно свидетельствовать о более активных процессах утраты функционально активной ткани щитовидной железы и уменьшении аутоиммунных реакций.

Ключевые слова: клинико-лабораторные показатели, артериальная гипертензия, первичный гипотиреоз, лечение. 


\title{
G.A. Ignatenko, I.V. Mukhin, T.S. Ignatenko, N.Yu. Panieva
}

\author{
SEI HPE «M. Gorky Donetsk National Medical University», Donetsk
}

\section{DYNAMICS OF CLINICAL AND LABORATORY MANIFESTATIONS OF THYROID SYNDROME IN HYPERTENSIVE PATIENTS WITH PRIMARY HYPOTHYROIDISM IN THE BACKGROUND OF TREATMENT}

One of the promising areas of non-drug treatment of this category of patients is the course use of hypoxyhyperoxytherapy sessions, which allows beneficial activation of the manifestations of hypertensive syndrome through the activation of NO-mediated endothelium-dependent vasodilation. However, the effect of hypoxy-hyperoxytherapy on thyroid metabolism in this category of patients remains poorly understood.

The aim of the study was to analyze the dynamics of the clinical and laboratory manifestations of thyroid syndrome in hypertensive patients with primary hypothyroidism against the background of two treatment regimens (only medication and similar, but with hypoxy-hyperoxytherapy).

The study included 95 patients with primary hypothyroidism aged 30 to 65 years. By random sampling, patients are divided into two homogeneous observation groups, statistically homogeneous by sex, age, duration of hypertensive and thyroid syndromes, the first of which $(\mathrm{n}=48)$ received only standard therapy (levothyroxine, angiotensin converting enzyme inhibitor / sartan, thiazide / thiazide-like diuretic, prolonged calcium antagonist, statin, antiplatelet agent). The second group ( $n=47)$, in addition to the same drug treatment, received hypoxichyperoxytherapy sessions ("Tibet-4", "Newlife", Russia). Hypoxy-hyperoxytherapy was carried out quarterly for 20 days each for 5 years (a total of 20 courses).

According to the results of the study, the initial clinical manifestations of thyroid syndrome in hypertensive patients with primary hypothyroidism were characterized by the prevalence of fatigue, paraorbital edema, de- pression, decreased memory, a feeling of chilliness, increased body weight and dry skin.

Comprehensive drug-hypoxy-hyperoxytherapy in group 2 was characterized by a significantly more intense decrease in the frequency of symptoms of thyroid syndrome than standard therapy in group 1 . The regression of symptoms of thyroid syndrome in group 2 at the 2nd stage of the examination compared to group 1 concerned such manifestations as: fatigue (-17.7\%), a feeling of chilliness $(-7.1 \%)$, an increase in body weight (-11.9\%). Moreover, in group 2 compared with group 1, the number of asymptomatic patients increased by $5.8 \%$, which indicates the transformation of hypothyroidism into the phase of sub- / compensation of thyroid metabolism.

The initial laboratory parameters of thyroid syndrome indicate the formation of primary hypothyroidism of moderate severity, frolic against the background of chronic autoimmune thyroiditis.

Treatment in group 2 contributed to the intensification of restoration of the level of thyroid hormones against the background of inhibition of thyroid stimulating hormone synthesis, which indicates drug compensation of thyroid metabolism. In contrast, in group 1, thyroid metabolism compensation was not received.

A more intense decrease in antithyroid antibody levels in group 1 may indirectly indicate more active processes of the loss of functionally active thyroid tissue and a decrease in autoimmune reactions.

Key words: clinical and laboratory parameters, arterial hypertension, primary hypothyroidism, treatment.

\section{ЛИТЕРАТУРА}

1. Братик А.В., Цыганова Т.Н. Эффективность интервальной гипоксической тренировки в медицине и спорте. Вестник новых медицинских технологий. 2013;1: 1218.

2. Вахитова3.Р., МухетдиноваГ.А., ФазыеваР.М. Сравнительная оценка факторов риска при артериальной гипертензии в сочетании с гипотиреозом. Практическая медицина. 2013; 6 (75): 163-166.

3. Вербовой А.Ф., ШарановаЛ.А., Косарева О.В., Вербовая Н.И., Долги Ю. А. Состояние сердечно-сосудистой системы при гипотиреозе. Клиническая медицина.2016; $94(7): 497-500$

4. Николаева А.Г. Использование адаптации к гипоксии в медицине и спорте. Витебск: ВГМУ; 2015: 150.

5. Самойлов В. О., Максимов А. Л., Филиппова Е. Б. и др. Влияние интервальных гипоксических тренировок на функциональное состояние человека в условиях гипоксической гипоксии. Вестник российской военномедицинской академии. 2014; 4 (48): 158-163.

6. Серебровская Т.В., Шатило В.Б. Опыт использования интервальной гипоксии для предупреждения и лечения заболеваний сердечно-сосудистой системы. Кровообращение и гемостаз. 2014; 1-2: 13-33.

7. Тишковец С.В., Нимаева Д.Э. Особенности лечения гипотиреоза. Вестник Бурятского государственного университета. 2016; 4: 23-29.

8. Третьяк С.И., Хрыщанович В.Я. Современные методы лечения гипотиреоза. Минск :БГМУ; 2011. 150.

\section{REFERENCES}

1. Bratik A.V., Tsyganova T.N. Effektivnost' interval'noi gipoksicheskoi trenirovki $\mathrm{V}$ meditsine i sporte. Vestnik novykh meditsinskikh tekhnologii. 2013;1: 12-18 (in Russian).

2. VakhitovaZ.R., MukhetdinovaG.A., FazyevaR.M. Sravnitel'naya otsenka faktorov riska pri arterial'noi gipertenzii v sochetanii s gipotireozom. Prakticheskaya meditsina. 2013; 6 (75): 163-166 (in Russian).

3. Verbovoi A.F., SharanovaL.A., Kosareva O.V., Verbovaya N.I., Dolgi Yu. A. Sostoyanie serdechno-sosudistoi sistemy pri gipotireoze. Klinicheskaya meditsina.2016; 94 (7): 497-500 (in Russian).

4. Nikolaeva A.G. Ispol'zovanie adaptatsii k gipoksii v meditsine i sporte. Vitebsk: VGMU; 2015: 150 (in Russian).

5. Samoilov V. O., Maksimov A. L., Filippova E. B. i dr. Vliyanie interval'nykh gipoksicheskikh trenirovok na funktsional'noe sostoyanie cheloveka v usloviyakh gipoksicheskoi gipoksii. Vestnik rossiiskoi voenno-meditsinskoi akademii. 2014; 4 (48): 158-163 (in Russian).

6. Serebrovskaya T.V., Shatilo V.B. Opyt ispol'zovaniya interval'noi gipoksii dlya preduprezhdeniya i lecheniya zabolevanii serdechno-sosudistoi sistemy. Krovoobrashchenie i gemostaz. 2014; 1-2: 13-33 (in Russian).

7. Tishkovets S.V., Nimaeva D.E. Osobennosti lecheniya gipotireoza. Vestnik Buryatskogo gosudarstvennogo universiteta. 2016; 4: 23-29 (in Russian).

8. Tret'yak S.I., Khryshchanovich V.Ya. Sovremennye metody lecheniya gipotireoza. Minsk :BGMU; 2011. 150 (in Rus- 
9. Феськова А.А., Дробышева Е.С., Овсянников Е.С., Чернов А.В. Влияние субклинической гипофункции щитовидной железы на клиническое течение гипертонической болезни / Прикладные информационные аспекты медицины. 2015; 6: 35-43.

10. Цыганова Т.Н., Прокопов А.Ф. Научные основы использования метода гипо-гиперокситерапии в практике митохондриальной медицины. Физиотерапевт. 2016; 3: 15-22

11. Danzi S.,Klein I. Thyroid disease and the cardiovascular system. Endocrinol. Metab. Clin. N. Am. 2014; 43, 2.: 517528.

12. Hofstetter L.,MesserliF.H. Hypothyroidism and hypertension: fact or myth? Lancet. 2018; 6, 391 (10115): 2930.

13. Stamatouli A., Bedoya P., Yavuz S. Hypothyroidism: Cardiovascular Endpoints of Thyroid Hormone Replacement. Front Endocrinol. 2020; 9, 10: 888-889. sian).

9. Fes'kova A.A., Drobysheva E.S., Ovsyannikov E.S., Chernov A.V. Vliyanie subklinicheskoi gipofunktsii shchitovidnoi zhelezy na klinicheskoe techenie gipertonicheskoi bolezni / Prikladnye informatsionnye aspekty meditsiny. 2015; 6: 35-43 (in Russian).

10. Tsyganova T.N., Prokopov A.F. Nauchnye osnovy ispol'zovaniya metoda gipo-giperoksiterapii v praktike mitokhondrial'noi meditsiny. Fizioterapevt. 2016; 3: 1522 (in Russian).

11. Danzi S.,Klein I. Thyroid disease and the cardiovascular system. Endocrinol. Metab. Clin. N. Am. 2014; 43, 2.: 517528.

12. Hofstetter L.,MesserliF.H. Hypothyroidism and hypertension: fact or myth? Lancet. 2018; 6, 391 (10115): 29-30.

13. Stamatouli A., Bedoya P., Yavuz S. Hypothyroidism: Cardiovascular Endpoints of Thyroid Hormone Replacement. Front Endocrinol. 2020; 9, 10: 888-889. 